Pacific Journal of Mathematics

ON CONJUGATION COBORDISM 


\section{ON CONJUGATION COBORDISM}

\section{DuAne O’NeILL}

An almost-complex manifold supports an involution if there is a differentiable self-map on the manifold of period two. The differential of the map acts on the coset space of the almost-complex structures on $M$ by inner automorphism. This action is also of period two. If the almost-complex structure is sent to its conjugate, the manifold with structure, together with the given involution is called a conjugation. Any linear involution of Euclidean space may be used to stabilize this situation, giving a cobordism theory of exotic conjugations. The question considered here is: What is the image in complex cobordism of the functor which forgets equivariance. The result shown in the next section is: If a stably almost-complex manifold supports an exotic conjugation, every characteristic number is even.

The first cobordism results on conjugations are due to Conner and Floyd [3] (\$24). In [4], Landweber established the equivariant analogues of the Thom theorems. Certain examples have been considered by Landweber, [5] (§3), and together with the result here the image of the forgetful functor can be seen to be maximal, in some cases.

2. Proof of the theorem. It is well-known from the work of Thom and Milnor that the unoriented bordism ring $\mathscr{N}_{*}$, with spectrum $M O$, is a polynomial ring over $Z_{2}$ on manifold classes $n_{t}$, $t+1$ any positive integer not a power of two ( $t$ nondyadic). Also $\mathscr{U}_{*}$, the complex bordism ring with spectrum $\boldsymbol{M U}$, is a polynomial ring over $Z$ on manifold classes $u_{t}, t=0,1, \cdots$. Representatives for the dyadic generators $u_{t}, t+1=2^{j}$, may be chosen so that every normal characteristic number is even. The principal ideal in $\mathscr{U}_{*}$ generated by dyadic generators is the graded Milnor ideal associated to $2, I$. $I_{2 k}=I \cap \mathscr{U}_{2 k}$.

If a partition of $k$ contains a dyadic integer the partition will be called dyadic. Let $d(k)$ denote the dyadic partitions of $k, n(k)$ the nondyadic partitions of $k$. If $\alpha=a_{1} a_{2} \cdots a_{r}$ is a partition of $k$ then the group generator $u_{a_{1}} \cdots u_{a_{r}} \in \mathscr{C}_{2 k}$ will be denoted $u_{\alpha}$. Similarly for $n_{\alpha} \in \mathscr{N}_{k}$.

If $M U(n)$ is given the involution defined in [4] then it is a $G$-complex, $G=Z_{2}$, in the sense of Bredon. Note that $\tilde{\omega}_{0}(M U(n))=$ $\tilde{\omega}_{1}(M U(n))=0$. The construction given in the next section produces, for each partition of $k, \alpha$, and sufficiently large $n$, an equivariant 
inclusion and a $G$-complex $e^{\alpha}: M U(n) \rightarrow Y^{\alpha}$ such that

(c i ) $\tilde{\omega}_{n+k}\left(Y^{\alpha}\right)=\left\{\begin{array}{cl}\left(Z_{2} \rightarrow 0\right) & \text { if } \alpha \in n(k) \\ 0 & \text { if } \alpha \in d(k)\end{array}\right.$

(c ii) $\tilde{\omega}_{2 n+2 k}\left(Y^{a}\right)=\left(0 \rightarrow\left\{Z,(-1)^{n+k}\right\}\right)$

(c iii) $\omega_{t}\left(Y^{\alpha}\right)=0$ if $t \neq n+k, 2 n+2 k$

(c iv) $e^{\alpha}\left(\frac{\boldsymbol{G}}{e}\right)_{\sharp}: \tilde{\boldsymbol{\omega}}_{2 n+2 k}(M U(n))\left(\frac{\boldsymbol{G}}{e}\right) \cong \mathscr{U}_{2 k} \rightarrow \tilde{\boldsymbol{\omega}}_{2 n+2 k}\left(Y^{\alpha}\right)\left(\frac{\boldsymbol{G}}{e}\right) \cong \boldsymbol{Z}$ maps $u_{\alpha}$ to an odd multiple of the generator $\alpha \in n(k)$.

Let the $r+s$ sphere with the orthogonal involution fixing an equatorial $s$-sphere be denoted $S^{r, s}$. The $G$-complex formed by attaching the cone over $S^{0, s}$ in $S^{r, s}$ will be denoted $S^{r, s} / S^{0,8}$. Let the equivariant homotopy groups

$$
\left|\left[\frac{S^{n+a, n+b}}{S^{0, n+b}}, M U(n)\right]\right| \text { and }\left|\left[\frac{S^{n+a, n+b}}{S^{0, n+b}}, Y^{\alpha}\right]\right|
$$

be denoted $\lambda \mathscr{C}_{a, b}$ and $\lambda Y_{a, b}$ respectively. It is understood that $a+b$ is much less than $n$ whenever this is used.

It is easy to see, from the cochain complex, [1] I $\S 6$, of $S^{r, s} / S^{0, s}$ that if $\tilde{\omega}$ is any generic coefficient system with a $G$-action $g$ on $\tilde{\omega}\left(\frac{G}{e}\right)$ then

$$
H_{G}^{k}\left(\frac{S^{r, s}}{S^{0, s}} ; \tilde{\omega}\right) \cong\left\{\begin{array}{cl}
0 \quad \text { if } 0<k \leqq s \quad \text { or } & r+s<k \\
\frac{\operatorname{Ker}\left(1+(-1)^{k-s} g\right)}{\operatorname{Im}\left(1+(-1)^{k-s-1} g\right)} & \text { if } s<k<r \\
\tilde{\omega}\left(\frac{G}{e}\right) & \text { if } k=r+s .
\end{array}\right.
$$

Note that the groups $\lambda Y_{a, b}$ are the same for all partitions $\alpha$ of $k$. I.e., by Bredon's classification theorem [1] II (2.11)

$$
\begin{aligned}
\lambda Y_{k+q, k-q} & \cong \frac{Z}{\left(1+(-1)^{q+1}\right) \boldsymbol{Z}} \\
\lambda Y_{k+q+t, k-q} & \cong\left\{\begin{array}{lll}
0 & q \text { even } \\
\boldsymbol{Z}_{2} & q \text { odd }
\end{array} \geqq 1\right. \\
\lambda Y_{l, m} & =0 \quad l+m<2 k .
\end{aligned}
$$

From this computation the main result may now be deduced. Let $\psi$ denote the forgetful functor.

THEOREM. $\quad u_{\alpha} \in$ Image $\left\{\psi: \lambda U_{k+q, k-q} \rightarrow \mathscr{U}_{2 k}\right\}$ only if $\alpha \in d(k)$.

Proof. Suppose $u_{\alpha}$ is in the image of $\psi$. Consider the com- 
mutative diagram with exact row (see [3], p. 286 for definitions of $\alpha, \beta$, and $\psi)$ :

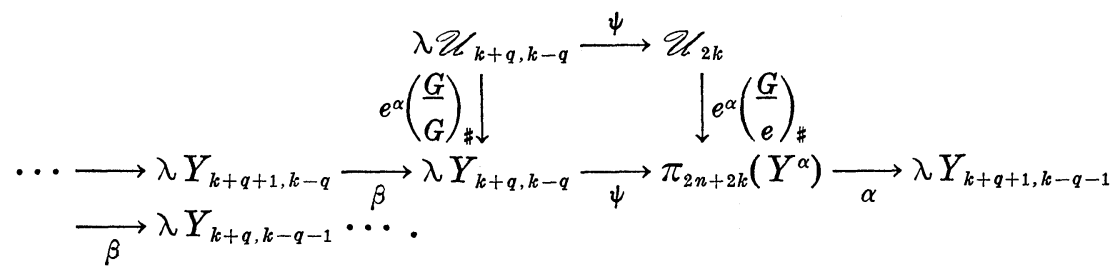

If $q$ were odd, the lower $\psi$ is zero. By (c iv) the upper $\psi$ is zero and $u_{\alpha}=0$, a contradiction. Now suppose $q$ is even. The exact row then is $0 \rightarrow \boldsymbol{Z} \rightarrow \boldsymbol{Z} \rightarrow \boldsymbol{Z}_{2} \rightarrow 0$ so that $e^{a}\left(\frac{\boldsymbol{G}}{e}\right)_{\sharp}$ maps $u_{\alpha}$ to an even multiple of the generator and by (c iv), $\alpha \in d(k)$.

COROLlaRY. Image $\psi \subseteq I$.

Proof. By ([4], (4.1)), $2 u_{\alpha} \in$ Image $\psi$ for every $\alpha$.

Then if $w \in$ Image $\psi$, subtract off even multiples of group generators until we have $w=2 w^{\prime}+u_{\alpha_{1}}+u_{\alpha_{2}}+\cdots+u_{\alpha_{l}}$. Now construct diagram (2.1) for $\alpha$ successively equal to $\alpha_{1}, \cdots, \alpha_{l}$. This shows that $\alpha_{1} \in d(k), \cdots, \alpha_{l} \in d(k)$, and the corollary is proved.

As a corollary of the construction in [5] $\S 3$ there are free exotic conjugations on representatives $u_{t}, t=2^{j}-1$, showing that Image $\left\{\psi: \lambda \mathscr{U}_{t+q, t-q} \rightarrow \mathscr{U}_{2 t}\right\}$ contains $u_{t}$ provided $q$ divisible by $2^{\phi(t+2)}$. Since the image of a forgetful functor is an ideal in $\mathscr{U}_{*}$ this shows:

COROLLARY. Image $\left\{\psi: \lambda U_{k+q, k-q} \rightarrow \mathscr{U}_{2 k}\right\}=I_{2 k}$ if $t=2^{j}-1 \leqq k<$ $2^{j+1}-1$ and $q$ divisible by $2^{\phi(t+2)} . \phi(m)$ is the familiar number equal to the number of integers $s, 0<s<m$ with $s \equiv 0,1,2,4(\bmod 8)$.

3. The construction. Recall Bredon's procedure for killing the homotopy groups of a $G$-space $X$, with $\tilde{\omega}_{0}\left(X, x_{0}\right)=\tilde{\omega}_{1}\left(X, x_{0}\right)=0$. Let $T$ be some $G$-set and $F(T)$ the free abelian $G$-module on $T$ such that Hom $\left(F(T), \tilde{\omega}_{r}(X)\right)$ contains an epimorphism $A_{r}$. By use of [2], Chapter II, (2.11), take a representative $a_{r}: S^{r}\left(T^{+}\right) \rightarrow X$ and define $X_{r+1}$ by the equivariant Puppe sequence,

$$
S^{r}\left(T^{+}\right) \stackrel{a_{r}}{\longrightarrow} X \stackrel{j}{\longrightarrow} X_{r+1} \longrightarrow S^{r+1}\left(T^{+}\right) \longrightarrow \cdots .
$$

Bredon shows, [2], (6.6), that

$$
\begin{aligned}
& j_{\ddagger}: \tilde{\omega}_{t}(X) \longrightarrow \tilde{\omega}_{t}\left(X_{r+1}\right) \text { is an isomorphism for } \\
& 0 \leqq t \leqq r-1 \text { and } \tilde{\omega}_{r}\left(X_{r+1}\right)=0 .
\end{aligned}
$$


In this construction of $Y^{\alpha}$ there are at most two $r$ where $A_{r}$ is not taken to be an epimorphism. To begin, let $\alpha$ be a partition of $k \geqq 0$ and take $n>2 k-1$ so that $\pi_{n+k}(M O(n))=\tilde{\omega}_{n+k}(M U(n))\left(\frac{G}{G}\right) \cong$ $\mathscr{N}_{k}$ and $\pi_{2 n+2 k}(M U(n))=\tilde{\omega}_{2 n+2 k}(M U(n))\left(\frac{G}{e}\right) \cong \mathscr{U}_{2 k}$. If $\alpha$ is dyadic let $n_{\alpha} \in \mathscr{N}_{k}$ denote the zero element. Regard $n_{\alpha}$ and $u_{\alpha}$ as elements of $\tilde{\omega}_{*}(M U(n))$.

Let $Y_{0}=M U(n)$ and let all $A_{r}$ be epimorphisms $0<r<n+k$. Denote the composition of the inclusions by $E_{r}: M U(n)=Y_{0} \subset \cdots \subset Y_{r}$. If $\alpha$ is dyadic, let $A_{r}$ be epimorphisms $0<r<2 n+2 k$; if not let $A_{n+k}$ be defined as follows. Let $T_{n+k}$ be the $G$-set of all elements in $\tilde{\boldsymbol{\omega}}_{n+k}\left(Y_{n+k-1}\right)\left(\frac{\boldsymbol{G}}{G}\right)$ except $E_{n+k \neq}\left(n_{\alpha}\right)$ and all elements in $\tilde{\boldsymbol{\omega}}_{n+k}\left(Y_{n+k-1}\right) \times\left(\frac{\boldsymbol{G}}{e}\right)$.

Take $A_{n+k}$ to be the natural homomorphism defined by extending the $G$-set inclusion $T_{n+k} \subseteq \tilde{\omega}_{n+k}\left(Y_{n+k-1}\right)$. Now let $A_{r}, n+k<r<$ $2 n+2 k$, be epimorphisms. Let the free cyclic summand containing $E_{2 n+2 k-1 *}\left(u_{\alpha}\right)$ in $\tilde{\omega}_{2 n+2 k}\left(Y_{2 n+2 k-1}\right)\left(\frac{G}{e}\right)$ be denoted $F$. Define $T_{2 n+2 k}$ to be the $G$-set of elements in the union of the sets $\tilde{\omega}_{2 n+2 k}\left(Y_{2 n+2 k-1}\right)\left(\frac{G}{G}\right)$ and $\tilde{\boldsymbol{\omega}}_{2 n+2 k}\left(Y_{2 n+2 k-1}\right)\left(\frac{G}{e}\right)-F$, and define $A_{2 n+2 k}$ to be the natural induced homomorphism. To define $Y_{r}, 2 n+2 k<r$, let $A_{r}$ be epimorphisms. This defines $Y^{\alpha}$ as a limit of $G$-complexes $M U(n)=Y_{0} \subset Y_{1} \subset \cdots$. Let $e^{\alpha}: M U(n) \rightarrow Y^{\alpha}$ be the inclusion.

It is clear that (c i) and (iii) are satisfied by this construction. To check the others some notation will be required. Let $g: S^{2 n+2 k} \rightarrow$ $M U(n)$ be some representative for $u_{\alpha}$, transverse regular on $B U(n) \subset$ $M U(n)$ and let $M_{\alpha}=g^{-1}(B U(n))$. Let $v_{n} \in \widetilde{H}^{2 n}(M U(n) ; Z)$ denote the universal Thom class and $s_{\alpha} \in H^{2 k}(B U(n) ; Z)$ the symmetric function associated to $\alpha$ in the universal Chern classes $c_{1}, c_{2}, \ldots$. Let $f: M U(n) \rightarrow$ $K(Z, 2 n+2 k)$ represent $s_{\alpha} \cup v_{n} \in \widetilde{H}^{2 n+2 k}(M U(n) ; Z)$. It is well-known that the degree defined by $f \circ g$ is the normal characteristic number of $M_{\alpha}, s_{\alpha}\left(u_{\alpha}\right)$.

The $G$-action of conjugation sends $c_{1}$ to $-c_{1}$, so by the splitting principle $c_{n}$ is sent to $(-1)_{c_{n}}^{n}, v_{n}$ to $(-1)^{n} v_{n}$ and $s_{\alpha} \cup v_{n}$ to $(-1)^{n+k} s_{\alpha} \cup$ $v_{n}$. However, this determines the $G$-action on homology which, through the Hurewicz isomorphism, gives the $G$-action on $\pi_{2 n+2 k}(M U(n))$. To check the remainder of (c ii) we attempt to extend the map $f$ to a map $h: Y^{\alpha} \rightarrow K(Z, 2 n+2 k)$.

The preceding construction shows that an extension of $f$ to $f^{\prime \prime}: Y_{2 n+2 k-1} \rightarrow K(Z, 2 n+2 k)$ exists for dimensional reasons. Thus there is an integer, $N \neq 0$, such that $N \cdot f_{\#}^{\prime \prime}\left(E_{2 n+2 k-1 \sharp}\left(u_{\alpha}\right)\right)=f_{\sharp}\left(u_{\alpha}\right)$ in $\pi_{2 n+2 k}(K(Z, 2 n+2 k))$. Note thatth is justifies the preceding claim that $E_{2 n+2 k-1 \ddagger}\left(u_{\alpha}\right)$ lies in an infinite cyclic summand in $\tilde{\omega}_{2 n+2 k}\left(Y_{2 n+2 k-1}\right)(G /(e$, 
$F$. Since $n+k$ may be taken odd, $F$ has only one fixed point, 0 . Thus, in the construction, Image $A_{2 n+2 k}$ and $F$ have only 0 in common. But $f_{\#}^{\prime \prime}$ lives on $F$, so an extension $f^{\prime}: Y_{2 n+2 k} K(Z, 2 n+2 k)$ exists. The desired extension, $h$, exists now by dimensional considerations and the following homotopy diagram commutes.

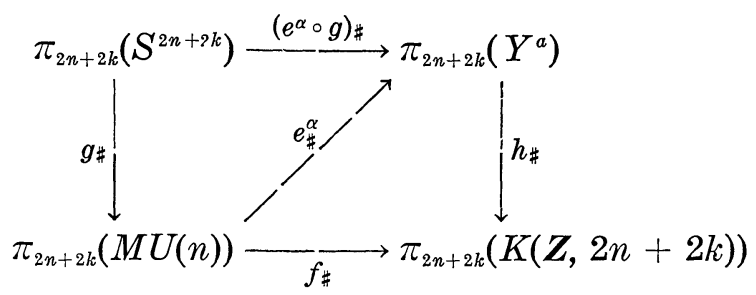

Since $f_{\#}$ carries a generator to nonzero multiple of the generator, $s_{\alpha}\left(u_{\alpha}\right) \cdot g$, we see that $\pi_{2 n+2 k}\left(Y^{\alpha}\right)$ cannot be finite. By construction, it is cyclic on one generator and this completes the verification of (c ii). From this diagram, note that $e_{\sharp}^{\alpha}$ carries $u_{\alpha}$ to some multiple of the generator, $y$, of $\pi_{2 n+2 k}\left(Y^{\alpha}\right), e_{\sharp}^{\alpha}\left(u_{\alpha}\right)=M y$. By commutativity, $M$ divides $s_{\alpha}\left(u_{\alpha}\right)$. But if $\alpha \in n(k), s_{\alpha}\left(u_{\alpha}\right)$ is odd; thus $M$ is odd and (c iv) is verified.

\section{REFERENCES}

1. G. E. Bredon, Equivariant Cohomology Theories, Lecture Notes in Math., (34), Springer-Verlag, 1967.

2. L Equivariant Homotopy, Proceedings of the Conference on Transformation Groups, Springer-Verlag, 1968.

3. P. E. Conner and E. E. Floyd, Differentiable Periodic Maps, Ergb. der Math., N. S. (33), Springer-Verlag, 1964.

4. P. S. Landweber, Conjugations on complex manifolds and equivariant homotopy of $M U$, Bull. Amer. Math. Soc., 74 (1968), 271-274.

5. _ Fixed point free conjugations on complex manifolds, Annals of Math., 86 (1967), 491-502.

Received September 19, 1973.

Suny at Buffalo 



\section{PACIFIC JOURNAL OF MATHEMATICS}

\section{EDITORS}

RICHARD ARENS (Managing Editor)

University of California

Los Angeles, California 90024

\section{J. DUGUNDJI}

Department of Mathematics University of Southern California Los Angeles, California 90007

D. Gilbarg and J. Milgram

Stanford University

Stanford, California 94305

University of Washington
Seattle, Washington 98105

ASSOCIATE EDITORS
E. F, BECKENBACH
B. H. NEUMANN
F. WOLF
K. Yoshida

\section{SUPPORTING INSTITUTIONS}

\author{
UNIVERSITY OF BRITISH COLUMBIA \\ CALIFORNIA INSTITUTE OF TECHNOLOGY \\ UNIVERSITY OF CALIFORNIA \\ MONTANA STATE UNIVERSITY \\ UNIVERSITY OF NEVADA \\ NEW MEXICO STATE UNIVERSITY \\ OREGON STATE UNIVERSITY \\ UNIVERSITY OF OREGON \\ OSAKA UNIVERSITY
}

\author{
UNIVERSITY OF SOUTHERN CALIFORNIA \\ STANFORD UNIVERSITY \\ UNIVERSITY OF TOKYO \\ UNIVERSITY OF UTAH \\ WASHINGTON STATE UNIVERSITY \\ UNIVERSITY OF WASHINGTON \\ * * * * \\ AMERICAN MATHEMATICAL SOCIETY \\ NAVAL WEAPONS CENTER
}

The Supporting Institutions listed above contribute to the cost of publication of this Journal, but they are not owners or publishers and have no responsibility for its content or policies.

Mathematical papers intended for publication in the Pacific Journal of Mathematics should be in typed form or offset-reproduced, (not dittoed), double spaced with large margins. Underline Greek letters in red, German in green, and script in blue. The first paragraph or two must be capable of being used separately as a synopsis of the entire paper. Items of the bibliography should not be cited there unless absolutely necessary, in which case they must be identified by author and Journal, rather than by item number. Manuscripts, in duplicate if possible, may be sent to any one of the four editors. Please classify according to the scheme of Math. Rev. Index to Vol. 39. All other communications to the editors should be addressed to the managing editor, or Elaine Barth, University of California, Los Angeles, California, 90024.

100 reprints are provided free for each article, only if page charges have been substantially paid. Additional copies may be obtained at cost in multiples of 50 .

The Pacific of Journal Mathematics is issued monthly as of January 1966. Regular subscription rate: $\$ 72.00$ a year (6 Vols., 12 issues). Special rate: $\$ 36.00$ a year to individual members of supporting institutions.

Subscriptions, orders for back numbers, and changes of address should be sent to Pacific Journal of Mathematics, 103 Highland Boulevard, Berkeley, California, 94708.

PUBLISHED BY PACIFIC JOURNAL OF MATHEMATICS, A NON-PROFIT CORPORATION

Printed at Kokusai Bunken Insatsusha (International Academic Printing Co., Ltd.), 270, 3-chome Totsuka-cho, Shinjuku-ku, Tokyo 160, Japan.

Copyright (C) 1973 by Pacific Journal of Mathematics Manufactured and first issued in Japan 


\section{Pacific Journal of Mathematics}

\section{Vol. 53, No. $2 \quad$ April, 1974}

Kenneth Abernethy, On characterizing certain classses of first countable spaces by

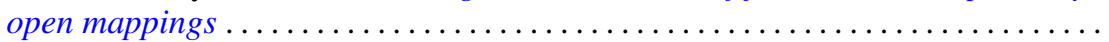

Ross A. Beaumont and Donald Lawver, Strongly semisimple abelian groups .......

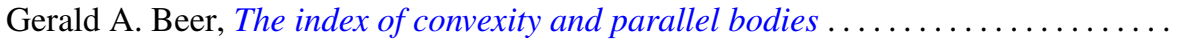

Victor P. Camillo and Kent Ralph Fuller, On Loewy length of rings ..............

Stephen LaVern Campbell, Linear operators for which $T^{*} T$ and $T T^{*}$ commute.

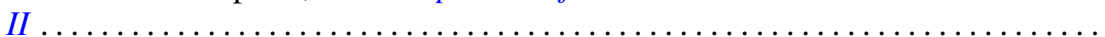

Charles Kam-Tai Chui and Philip Wesley Smith, Characterization of a function by

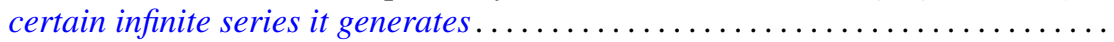

Allan L. Edelson, Conjugations on stably almost complex manifolds . ...........

Patrick John Fleury, Hollow modules and local endomorphism rings . . ..........

Jack Tilden Goodykoontz, Jr., Connectedness im kleinen and local connectedness in

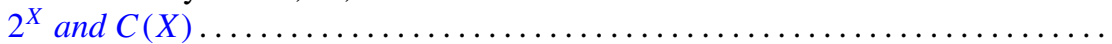

Robert Edward Jamison, II, Functional representation of algebraic intervals .......

Athanassios G. Kartsatos, Nonzero solutions to boundary value problems for

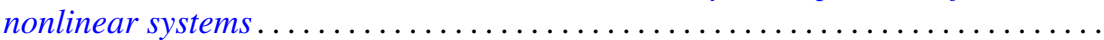

Soon-Kyu Kim, Dennis McGavran and Jingyal Pak, Torus group actions on simply

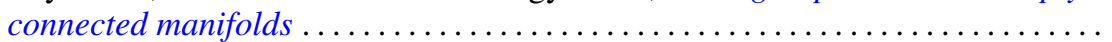

David Anthony Klarner and R. Rado, Arithmetic properties of certain recursively

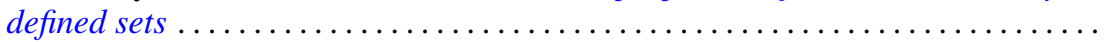

Ray Alden Kunze, On the Frobenius reciprocity theorem for square-integrable

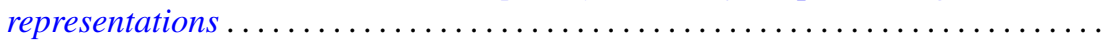

John Lagnese, Existence, uniqueness and limiting behavior of solutions of a class of differential equations in Banach space...

Teck Cheong Lim, A fixed point theorem for families on nonexpansive mappings Lewis Lum, A quasi order characterization of smooth continua

Andy R. Magid, Principal homogeneous spaces and Galois extensions . .

Charles Alan McCarthy, The norm of a certain derivation ..... . .

Louise Elizabeth Moser, On the impossibility of obtaining $S^{2} \times S^{1}$ by elementary surgery along a knot. .

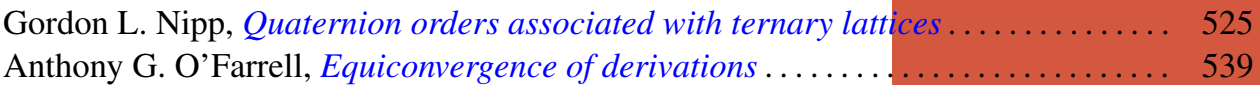

Dorte Olesen, Derivations of $A W^{*}$-algebras are inner . . . . . . . . . . . . . . . 555

Dorte Olesen and Gert Kjærgaard Pedersen, Derivations of $C^{*}$-algebras have

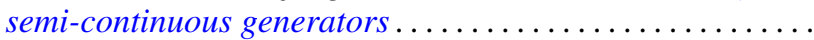

Duane O’Neill, On conjugation cobordism.

Chull Park and S. R. Paranjape, Probabilities of Wiener paths crossing differentiable

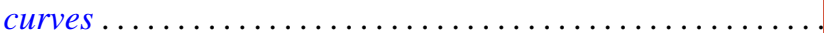

Edward Ralph Rozema, Almost Chebyshev subspaces of $L^{1}(\mu$;

Lesley Millman Sibner and Robert Jules Sibner, A note on the Atiyah-Bott fixed

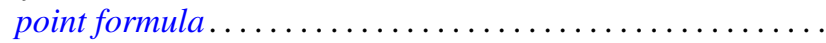

Betty Salzberg Stark, Irreducible subgroups of orthogonal groups generated by

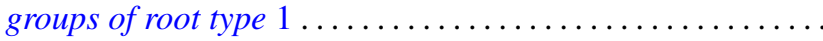

N. Stavrakas, A note on starshaped sets, $(k)$-extreme points and the half ray

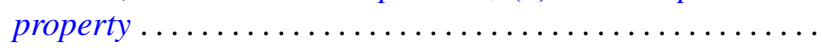

Carl E. Swenson, Direct sum subset decompositions of $Z \ldots \ldots$ 